\title{
Atypical respiratory distress in eastern Democratic Republic of the Congo prior to the COVID-19 pandemic. A case report
}

Guy-Quesney Mateso ${ }^{1 \dagger}$, Marius Baguma ${ }^{1,2^{*+}}$ (D) Pacifique Mwene-Batu ${ }^{1,2,3 \dagger}$, Ghislain Maheshe Balemba ${ }^{1,2,3}$, Fabrice Nzabara ${ }^{1,2}$, Samuel Makali ${ }^{1,3,2}$, Aline Bedha ${ }^{1,2}$, Bonheur Furaha' ${ }^{1}$, Jimmy Minani ${ }^{1}$, Christian Tshongo Muhindo 1,2, Espoir Bwenge Malembaka 2,3, Mannix Imani Masimango 1,2, Tony Akilimali Shindano ${ }^{1,2}$, Justin Cirhuza Cikomola ${ }^{1,2}$ and Kanigula Mubagwa ${ }^{2}$

\begin{abstract}
Background: Predictions have been made that Africa would be the most vulnerable continent to the novel Coronavirus disease 2019 (COVID-19). Interestingly, the spread of the disease in Africa seems to have been delayed and initially slower than in many parts of the world. Here we report on two cases of respiratory distress in our region before the official declaration of the disease in December 2019, cases which in the present times would be suspect of COVID-19.

Case presentation: These two cases (one 55-year-old man and one 25-year-old woman) of acute respiratory distress secondary to atypical pneumonia were seen in Bukavu, in Eastern Democratic Republic of the Congo (DRC), between September and December 2019. One patient had returned from China and the other had close contacts with travellers from China in the 2 weeks prior to the onset of symptoms. In either case, the aetiology could not be accurately determined. However, the two cases presented a clinical picture (progressive dyspnoea, preceded by dry cough and fever) and laboratory changes (procalcitonin within the normal range, slight inflammation, and lymphopenia) compatible with a viral infection. The chest X-ray series of the first patient showed lesions (reticulations, ground glass, and nodules $\leq 6 \mathrm{~mm}$ ) similar to those currently found in COVID-19 patients. In addition, unlike the 25-year-old female patient who had no comorbidity, the 55-year-old male patient who had hypertension as comorbidity, developed a more severe acute respiratory distress which progressed to death.

Conclusion: These cases bring to the attention the fact that COVID-19-like syndromes may have already been present in the region months before the official beginning of the pandemic. This also brings to question whether a prior presence of the disease or infections with related virus may account for the delayed and less extensive development of the pandemic in the region.
\end{abstract}

Keywords: COVID-19, SARS-Cov-2 coronavirus, Pandemic, Democratic Republic of the Congo, DRC, Case report

\footnotetext{
* Correspondence: baguma.akonkwa@ucbukavu.ac.co

${ }^{\dagger}$ Guy-Quesney Mateso, Marius Baguma and Pacifique Mwene-Batu contributed equally to this work.

'Department of Internal Medicine, Hôpital Provincial Général de Référence

de Bukavu (HPGRB), Bukavu, Democratic Republic of the Congo

${ }^{2}$ Faculty of Medicine, Université Catholique de Bukavu (UCB), Bukavu,

Democratic Republic of the Congo

Full list of author information is available at the end of the article
}

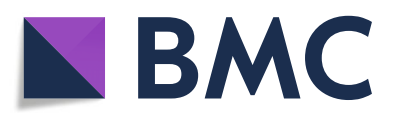

(- The Author(s). 2021 Open Access This article is licensed under a Creative Commons Attribution 4.0 International License, which permits use, sharing, adaptation, distribution and reproduction in any medium or format, as long as you give appropriate credit to the original author(s) and the source, provide a link to the Creative Commons licence, and indicate if changes were made. The images or other third party material in this article are included in the article's Creative Commons licence, unless indicated otherwise in a credit line to the material. If material is not included in the article's Creative Commons licence and your intended use is not permitted by statutory regulation or exceeds the permitted use, you will need to obtain permission directly from the copyright holder. To view a copy of this licence, visit http://creativecommons.org/licenses/by/4.0/. The Creative Commons Public Domain Dedication waiver (http://creativecommons.org/publicdomain/zero/1.0/) applies to the data made available in this article, unless otherwise stated in a credit line to the data. 


\section{Background}

The world is currently facing a devastating pandemic of the novel coronavirus disease 2019 (COVID-19), caused by severe acute respiratory syndrome coronavirus 2 (SARS-CoV-2), which was first declared in Wuhan, China, in December 2019 [1]. Clinically, the infection by the virus is asymptomatic in most cases $[2,3]$. In many cases, it may manifest itself by non-typical symptoms such as fever, cough, sore throat [4-8], known to be present in common cold. In some other cases more evocative symptoms such as anosmia and ageusia may be present [9-12]. Most severe cases develop respiratory distress with hypoxia $[5,6]$. Laboratory findings include an inflammatory syndrome, mild lymphopenia and an increased level of cytokines [13]. Pneumonia is present on chest X-rays, and more specific aspects such as ground-glass images can be detected on radiography or CT scan [5-8]. Although the first COVID-19 cases were described in China [4-8], the spread of the disease in other countries and regions, such as Western Europe, United States, South America (e.g., Brazil), or South Africa has been higher than in China [14]. As of February 22, 2021, over 111 million cases of COVID-19 have been confirmed in 192 countries, including more than 2.46 million deaths $[14,15]$. Predictions have been made that Africa would be the most vulnerable continent to COVID-19, because of various factors: dense population in many cities, close physical contacts indoors due to poor housing conditions or outdoors due to the fact that many people rely on daily income from small business, high prevalence of chronic diseases (e.g., infections by human immunodeficiency virus (HIV), malnutrition, etc.) interfering with the immune defence, absent or poor infrastructures to diagnose or treat patients, and very frequent travels between China and Africa $[16,17]$. Sub-Saharan African countries are shown to have the world's highest estimated vulnerability index to infectious disease outbreaks [18]. The Democratic Republic of the Congo (DRC) is considered to be among the countries at highest risk, especially since travel continued even after the pandemic was declared [16].

Interestingly, except for South Africa, the spread of the disease in the rest of sub-Saharan Africa seems to have been delayed and occurred to a relatively smaller extent than in many parts of the world. Since the first COVID-19 case was officially identified in Africa (in Egypt) on February 14, 2020 [19, 20], 2,780,836 confirmed cases and 70,107 deaths have been documented as of February 21, 2021, about 1 year later [14]. These numbers are far below those recorded in other parts of the world. In the DRC, the first case has been reported on March 10, 2020, in Kinshasa (12 million inhabitants), but as of February 19, 2021, only 19,047 confirmed cases have been registered in the same city and 25,080 countrywide [21]. In Bukavu (about 1 million inhabitants, density: 16,600 inhabitants $/ \mathrm{km}^{2}$ ), the capital city of South-Kivu, in Eastern DRC, the first two COVID-19 cases were travellers who arrived on March 18 and 20, 2020 , but despite a delay in quarantine measures (started on March 30,2020), nearly 1 year later the province has registered only 726 confirmed cases of which 56 were deaths [22].

The reasons for the low rate of COVID-19 propagation in Africa remain unknown. Recent data suggest a relatively high level of infection with other coronaviruses $[23,24]$. Although it remains unclear whether such infections are associated with cross-immunity and increased resistance to COVID-19, it is of interest to document and test cases of severe respiratory distress presenting with symptoms like those of COVID-19. In this report, we present two patients who were admitted between September and December 2019 for acute respiratory distress in the Intensive Care Unit (ICU) of the "Hôpital Provincial Général de Référence de Bukavu" (HPGRB), in Bukavu. For both patients, a diagnosis of atypical viral pneumonia was made. COVID-19 was not yet known at that time; hence the patients were not tested for the disease, but the clinical presentation, radiological findings, and the course of the disease are similar to those of COVID-19.

Our report is timely in that it coincides with a moment the debate about herd immunity is rife in many countries in Europe and North America which are grappling with successive waves of COVID-19 and societal impacts of containment measures. Documenting the presence of other viral infections on the African continent way longer before the COVID-19 pandemic unveiled, may help understand the seemingly downward trends in the COVID-19 spread on the continent, which contrasts with the severe damages (higher numbers of hospitalisations, intensive care admissions, and deaths) as in Europe and the Americas. Our report also comes to further underpin the urgent call for inclusion of Africa in the current planning and prioritization processes for global COVID-19 research [25].

\section{Methods}

Upon learning of coronavirus, we revisited the files of a few patients who had been hospitalized during the last term of 2019, and enrolled the two patients presented in this report, who had been admitted with fever, cough, and hypoxia with signs of atypical pneumonia on chest radiography.

We reviewed their medical records to collect information on physical examination, and the laboratory (including haematology, biochemistry, and microbiology tests) as well as the chest X-ray findings. Complete blood count was carried out using an automated haematology 
analyser (Edan H50, Hamburg, Germany). Biochemical tests on plasma or serum were done using semiautomatic analysers (CYANSmart CY009, CYPRESS Diagnostics, Langdorp, Belgium, for creatinine and blood urea nitrogen; Rayto Chemray 120, Shenzhen, China, for alanine transaminase, aspartate aminotransferase, alkaline phosphatase, albumin, and bilirubin; iCHROMA II, Brussels, Belgium, for D-dimer and procalcitonin; EDAN i15 analyser, Hamburg, Germany, for arterial blood gas). Bacteriological tests included blood cultures performed in BACT/ALERT ${ }^{\circ}$ FA Plus and BACT/ALERT ${ }^{\circ}$ FN culture media (bioMerieux, Marcy-l'Etoile, France), screening of tuberculosis using a Ziehl-Neelsen stain of sputum, and microscopic examination of Giemsa-stained thick blood film for diagnosis of clinical malaria. At the time of the hospitalization of the two patients, there was no capacity for regular testing for the presence of respiratory virus. HIV test was carried out on serum samples using rapid point-of-care test Determine ${ }^{\mathrm{Tw}} \mathrm{HIV}-1 / 2$ $\mathrm{Ag} / \mathrm{Ab}$ Combo (Alere Inc., Florida, USA) which detects HIV antigens as well as antibodies [26]. Chest X-rays were carried out using Perlong PL 50DR Digital Radiography (Nanjing, China).

No ethical permission was needed since the study involved a retrospective review of data from already treated patients, whose identity was kept confidential.

\section{Case presentation \\ Case 1}

A 55-year-old man, with a 10-year history of hypertension (treated with nicardipine $20 \mathrm{mg}$ twice daily, bisoprolol $5 \mathrm{mg}$ twice daily and aldactazine 1 tablet once daily) and a 5-year history of pigeon breeding, was seen in the Department of Internal Medicine on September 20, 2019, for dry cough, 2 weeks after returning from Guangzhou (Guangdong province, China). The patient did not complain of any loss of smell or taste. He had good mental orientation. Axillary temperature was $37^{\circ} \mathrm{C}$. Chest auscultation noted normal heart sounds and normal vesicular breath sounds without rales or sibilance. There was no abdominal tenderness nor enlarged liver or spleen. There was no low limb oedema. There was no loss of peripheral motor or sensor function and no other abnormal neurological signs. Laboratory results showed normal complete blood count $(\mathrm{CBC})$ and procalcitonin $(<0.1 \mathrm{ng} / \mathrm{ml})$; an elevated serum creatinine $(1.8 \mathrm{mg} / \mathrm{dl}$, hence a glomerular filtration rate, GFR, of $50.6 \mathrm{ml} /$ $\left.\left(\min ^{*} 1.73 \mathrm{~m}^{2}\right)\right)$ and electrolytes disturbances (low sodium at $130 \mathrm{mmol} / \mathrm{l}$, low potassium at $3.4 \mathrm{mmol} / \mathrm{l}$ and low calcium $1.04 \mathrm{mmol} / \mathrm{l}$ ) (Table 1). A diagnosis of non-specific interstitial pneumonia was evoked based on chest X-rays showing bi-basal and posterior densification (25 to 30\% of total chest height), blurring heart borders and diaphragm, associated with trabecular bands and ground- glass zones on the mid third of the lungs (Fig. 1a, b). He was sent back home with oral azithromycin $(500 \mathrm{mg}$ daily for 5 days), paracetamol (1000 $\mathrm{mg}$ up to 3 times a day if necessary) and an antitussive drug.

Despite treatment, coughing was exacerbated, and fever and dyspnoea occurred 2 weeks later. On October 10, 2019, he was admitted in the Emergency ward. The clinical evaluation noticed polypnea ( 30 breaths $/ \mathrm{min}$ ), tachycardia (100 beats/min) and fever $\left(38^{\circ} \mathrm{C}\right)$. Oxygen saturation $\left(\mathrm{SaO}_{2}\right)$ measured with a pulse oximeter was 54\% [normal range: $95-100 \%$ ]. The patient presented a respiratory distress (nose flaring, chest retractions and cyanosis) and had fine crackles at both lung bases. Cardiovascular examination was normal. Laboratory results showed a moderate inflammatory syndrome with hyper-

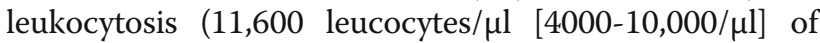
which 9071 neutrophils [1500-7000/ $\mu \mathrm{l}]$ and 1380 lymphocytes $[1500-4500 / \mu \mathrm{l}]$ ) and increased C-reactive protein (CRP, $54.9 \mathrm{mg} / \mathrm{l}[0-3 \mathrm{mg} / \mathrm{l}])$. Procalcitonin levels were normal [below $0.1 \mathrm{ng} / \mathrm{ml}$ ]. An arterial blood gas (ABG) analysis revealed the presence of severe hypoxemia $\left(\mathrm{P}_{\mathrm{a}} \mathrm{O}_{2} 33 \mathrm{mmHg}\right.$ [75-100 $\mathrm{mmHg}$ ], $\left.\mathrm{SaO}_{2} 74 \%\right)$ and respiratory alkalosis ( $\mathrm{pH} 7.55$ [7.35-7.45], $\mathrm{P}_{\mathrm{a}} \mathrm{CO}_{2} 29.7$ $\mathrm{mmHg}$ [35-45 mmHg], $\mathrm{P}_{\mathrm{a}} \mathrm{O}_{2} / \mathrm{FiO}_{2}$ ratio $157 \mathrm{mmHg}$ [400-500 $\mathrm{mmHg}]$, bicarbonate $25.1 \mathrm{mmol} / \mathrm{l}$ [22-26 $\mathrm{mmol} / \mathrm{l}]$, and lactates $1.89 \mathrm{mmol} / \mathrm{l}[<2 \mathrm{mmol} / \mathrm{l}])$. Bacteriological investigations were all negative. Tuberculosis was unlikely given a Ziehl-Neelsen stain of sputum which was negative for three samples collected at different times. HIV serological tests were negative (Table 1). A second chest X-rays showed persistence of the aforementioned findings, except for the trabeculae hidden by the alveolar densification that had progressed up to the level of the main bronchi, overhung by a well delimited ground glass zone without encroachment upon the apices (Fig. 1c).

The patient was hospitalized in the ICU for acute respiratory distress syndrome secondary to viral pneumonia with probable bacterial superinfection. He received oxygen by mask $(5 \mathrm{l} / \mathrm{min})$ and intravenous antibiotics (combination of amoxicillin and clavulanic acid 1/0.25 g thrice daily). Despite this treatment, continuous fever (average temperature: $38.5^{\circ} \mathrm{C}$ ) and hypoxemia persisted, and lactates increased to $4 \mathrm{mmol} / \mathrm{l}$. So, 2 days later, amoxicillin and clavulanic acid were replaced by levofloxacin $(500 \mathrm{mg})$ and ceftriaxone $(1 \mathrm{~g})$, both twice daily, in addition to intravenous dexamethasone $(16 \mathrm{mg}$ thrice daily).

Four days later, no improvement was observed. The oxygen flow was increased to $10 \mathrm{l} / \mathrm{min}$ and dexamethasone replaced by methylprednisolone (125 mg twice daily for 5 days). During the following week, there was a slight improvement of the dyspnoea and fever, but on October 23, 2019, fever reappeared, and the respiratory distress 
Table 1 Summary of laboratory tests for the 55-year-old male patient

\begin{tabular}{|c|c|c|c|c|c|c|c|}
\hline Laboratory tests & Normal values & Sept. 20 & Oct. 10 & Oct. 14 & Oct. 17 & Oct. 23 & Oct. 28 \\
\hline \multicolumn{8}{|l|}{ Arterial Blood Gas } \\
\hline $\mathrm{pH}$ & $7.35-7.45$ & - & 7.55 & 7.52 & 7.55 & 7.31 & 6.97 \\
\hline $\mathrm{PaO}_{2}(\mathrm{mmHg})$ & $75-100$ & - & 33 & 48 & 49 & 60 & 60 \\
\hline $\mathrm{PCO}_{2}(\mathrm{mmHg})$ & $35-45$ & - & 29.7 & 36.5 & 31.6 & 43 & 74 \\
\hline $\mathrm{SaO}_{2}(\%)$ & $94-100$ & - & 74 & 88 & 89 & 92 & 73 \\
\hline Lactates (mmol/L) & $<2.00$ & - & 1.89 & 4.00 & 3.27 & 3.1 & 12.2 \\
\hline Bicarbonates (mmol/L) & $22.0-26.0$ & - & 25.1 & 28.8 & 27.0 & 21 & 16.5 \\
\hline $\mathrm{P}_{\mathrm{a}} \mathrm{O}_{2} / \mathrm{FiO}_{2}(\mathrm{mmHg})$ & $400-500$ & - & 157 & 229 & 233 & 60 & 60 \\
\hline \multicolumn{8}{|l|}{ Complete blood count } \\
\hline White blood cells $\left(\times 10^{3} / \mu \mathrm{L}\right)$ & $4.00-10.00$ & 8.00 & 11.60 & - & - & - & 7.60 \\
\hline Neutrophils $\left(\times 10^{3} / \mu \mathrm{L}\right)$ & $1.50-7.00$ & 5.46 & 9.07 & - & - & - & 5.40 \\
\hline Lymphocytes $\left(\times 10^{3} / \mu \mathrm{L}\right)$ & $1.50-4.50$ & 1.63 & 1.38 & - & - & - & 1.43 \\
\hline Monocytes $\left(\times 10^{3} / \mu \mathrm{L}\right)$ & $0.20-1.00$ & 0.74 & 1.11 & - & - & - & 0.74 \\
\hline Eosinophils $\left(\times 10^{3} / \mu \mathrm{L}\right)$ & $0.10-0.50$ & 0.16 & 0.02 & - & - & - & 0.03 \\
\hline Red blood cells $\left(\times 10^{6} / \mu \mathrm{L}\right)$ & $4.20-5.70$ & 5.28 & 4.90 & - & - & - & 5.41 \\
\hline Haemoglobin (g/L) & 13.0-18.0 & 16.0 & 15.3 & 17.0 & - & - & 16.5 \\
\hline Haematocrit (\%) & $40.0-52.0$ & 45.1 & 42.3 & 50.0 & - & - & 48 \\
\hline Platelets $\left(\times 10^{3} / \mu \mathrm{L}\right)$ & $150-450$ & 244 & 213 & - & - & - & 156 \\
\hline \multicolumn{8}{|l|}{ Electrolytes } \\
\hline Sodium (mmol/L) & $135-155$ & 130 & 129 & 135 & 132 & - & 133 \\
\hline Potassium (mmol/L) & $3.5-5.1$ & 3.4 & 3.4 & 3.2 & 3.4 & - & 3.7 \\
\hline Chloride (mmol/L) & $96-115$ & 99 & 99 & 102 & 99 & - & 115 \\
\hline Calcium (mmol/L) & $1.10-1.40$ & 1.04 & 1.12 & 1.21 & 1.25 & - & 1.33 \\
\hline Magnesium (mg/dL) & $1.60-2.50$ & - & - & 1.8 & - & - & 1.7 \\
\hline C-Reactive protein (mg/L) & $<3.0$ & - & 54.9 & - & 98 & 43 & 102 \\
\hline Procalcitonin (ng/mL) & $<0.10$ & $<0.10$ & $<0.10$ & - & - & 0.69 & $<0.1$ \\
\hline Creatinine (mg/dL) & $0.6-1.40$ & 1.80 & 2.04 & - & - & 1.80 & 3.8 \\
\hline Blood Urea Nitrogen & $8.0-23.0$ & - & 40.0 & - & - & 107 & 150 \\
\hline Blood Glucose (mg/dl) & $60-110$ & 82 & 127 & - & - & 82 & - \\
\hline $\mathrm{Hb} 1 \mathrm{Ac}(\%)$ & $4-5.70$ & 3.29 & - & - & - & - & - \\
\hline Troponin (ng/ml) & & 0.02 & - & - & - & - & - \\
\hline Pro-BNP (pg/ml) & & - & 183.4 & - & - & 101.5 & - \\
\hline D-dimer ( $\mu \mathrm{g} / \mathrm{L})$ & $<500$ & - & 790 & - & - & - & - \\
\hline Thick blood film & Negative & - & Negative & - & - & Negative & - \\
\hline Haemocultures & Negative & - & Negative & - & - & Negative & - \\
\hline Ziehl-Neelsen stain of sputum & Negative & - & Negative & - & - & Negative & - \\
\hline \multicolumn{8}{|l|}{ Serologies } \\
\hline HIV & Negative & - & Negative & - & - & - & - \\
\hline Viral hepatitis B & Negative & - & Negative & - & - & - & - \\
\hline Viral hepatitis C & Negative & - & Negative & - & - & - & - \\
\hline Syphilis & Negative & - & Negative & - & - & - & - \\
\hline
\end{tabular}

worsened. A new chest X-ray showed a reduction of the alveolar consolidation to the advantage of an interstitial syndrome (reticulations, ground-glass, and nodules $\leq 6$ $\mathrm{mm}$ ) extending to lung tops (Fig. 1d). Meanwhile, the CRP was $49 \mathrm{mg} / \mathrm{l}$ and procalcitonin $0.62 \mathrm{ng} / \mathrm{ml}$. A diagnosis of respiratory zoonosis was considered, given the 

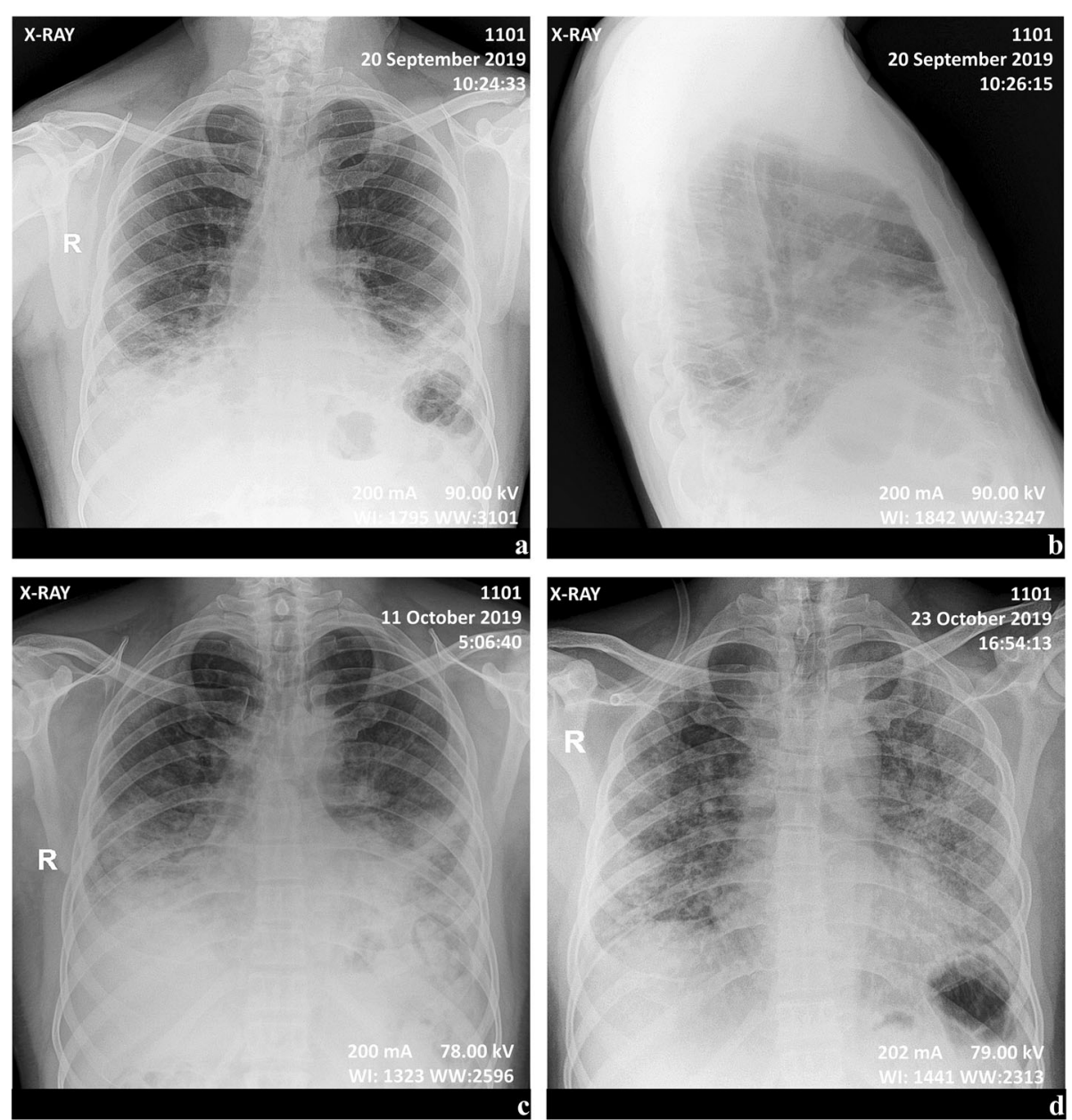

Fig. 1 Serial chest X-rays of Patient 1. Multifocal then diffuse airspace disease in a 55-years-old man. Bibasal lung consolidation with assorted trabeculae, especially in the posterior regions, overhung by discreet hazy opacities $(\mathbf{a}, \mathbf{b})$. Follow-up showing a cranially progress of the predominant inhomogeneous consolidation still coexisting with a clearly diffuse ground-glass (c). There is apparent replacement of alveolar densification by diffuse reticular interstitial marks with some few parahilar nodules (d)

history of pigeon breeding. Intravenous methylprednisolone (125 mg twice daily) was reintroduced and Duovent $^{\circ}$ (combined ipratropium and fenoterol) was administered in nebulization, without any improvement. $\mathrm{SaO}_{2}$ (measured with a pulse oximeter) remained at $40 \%, \mathrm{P}_{\mathrm{a}} \mathrm{O}_{2} / \mathrm{F}_{\mathrm{i}} \mathrm{O}_{2}$ ratio decreased to less than $70 \mathrm{mmHg}$, and the patient became confused, with loss of notion of space and time, probably because of the hypoxia.

On October 25, 2019, the patient underwent orotracheal intubation for mechanical ventilation. This improved the $\mathrm{SaO}_{2}$ to $90-94 \%$ but on October 28, 2019, the patient developed a shock, with increased lactacidemia $(12.2 \mathrm{mmol} / \mathrm{l})$, followed within a few hours by cardiac arrest irresponsive to resuscitation (Fig. 2).

\section{Case 2}

A 25-year-old woman, with no history of recent travel, was admitted at the Emergency ward on December 18,
2019, for a one-week progressive dyspnoea, preceded by dry cough and fever. She had no particular medical history and was a nurse in a hospital where Chinese employees from a multinational mining company are treated, of whom some had recently travelled from China (the place they came from in China could not be determined) and three of them had presented flu-like symptoms within 2 weeks. On admission, she could not complete sentences due to dyspnoea. At ambient air, she presented a $\mathrm{SaO}_{2}$ of $82 \%$ and signs of respiratory distress without cyanosis. Her pulmonary and cardiac auscultation was normal.

The chest X-rays showed reticular lines and peribronchovascular haziness in the infrahilar and retrocardiac regions, bilaterally. This suggested a mild interstitial pneumonia (Fig. 3a, b). Laboratory results showed a slight inflammation with CRP at $14.5 \mathrm{mg} / \mathrm{l}$, lymphopenia (700 lymphocytes/ $\mu \mathrm{l})$ and normal procalcitonin $(<0.1$ 


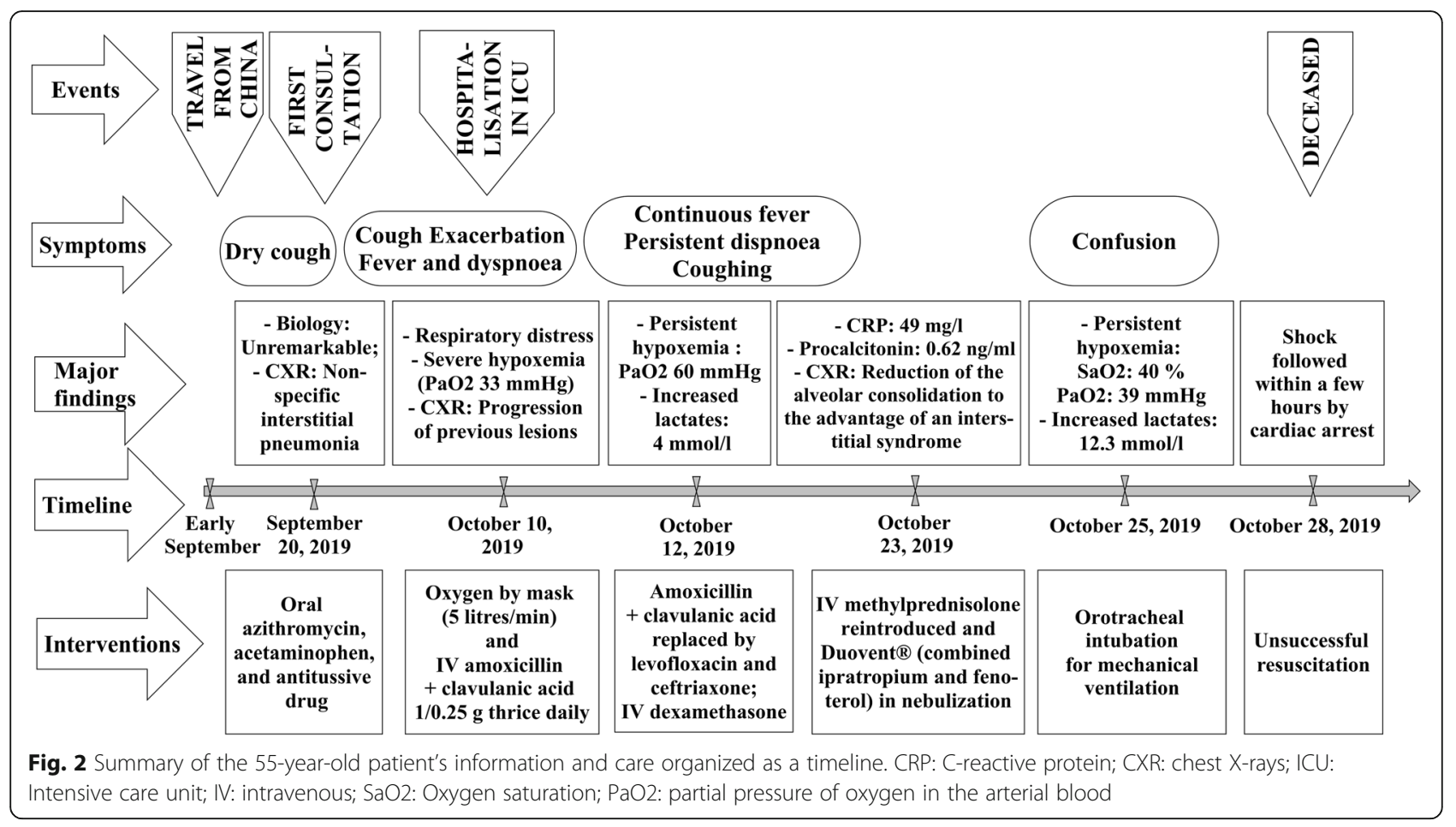

ng/ml). The ABG showed a hypoxemia $\left(\mathrm{P}_{\mathrm{a}} \mathrm{O}_{2} 60 \mathrm{mmHg}\right)$ and a respiratory alkalosis $\left(\mathrm{pH} 7.51, \mathrm{P}_{\mathrm{a}} \mathrm{CO}_{2} 35 \mathrm{mmHg}\right.$, $\mathrm{P}_{\mathrm{a}} \mathrm{O}_{2} / \mathrm{F}_{\mathrm{i}} \mathrm{O}_{2} 286 \mathrm{mmHg}$, bicarbonate $27.1 \mathrm{mmol} / \mathrm{l}$, and lactates $1.50 \mathrm{mmol} / \mathrm{l})$. Creatinine, blood urea nitrogen and blood electrolytes were normal. HIV serology was negative.

A diagnosis of moderate acute respiratory distress syndrome secondary to a viral pneumonia was retained and the patient was admitted in the ICU, receiving oxygen (4 l/min), azithromycin (500 $\mathrm{mg}$ once daily for 5 days) and Duovent ${ }^{\circ}$ in nebulization. Three days later, she was eupnoeic with normal $\mathrm{SaO}_{2}$ at ambient air. She was discharged from hospital 5 days after admission (Fig. 4).

\section{Discussion}

In this report, we present two cases of acute respiratory distress secondary to atypical pneumonia in eastern DRC. One patient had returned from China and the other had had close contacts with employees who had returned from a travel to China in the 2 weeks prior to the onset of symptoms. In one case, the respiratory disease did not respond to treatments for typical and
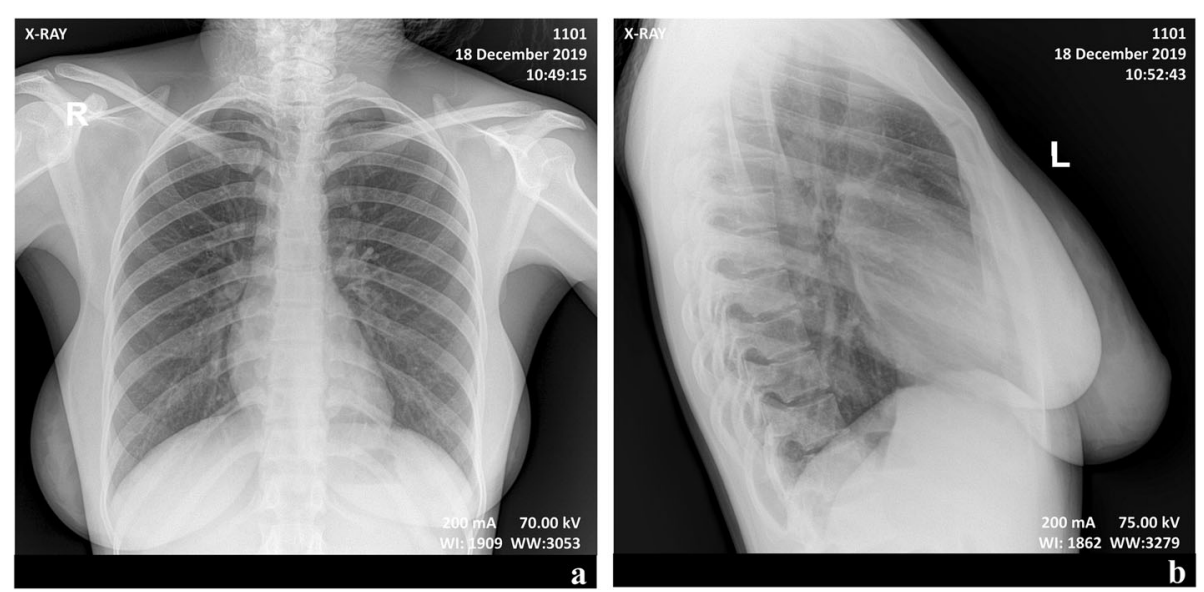

Fig. 3 Chest X-rays of Patient 2. Subnormal chest X-rays of a 25-year-old female. Discrete peribronchovascular blur in the posterobasal regions. Normal aspect of the hila and the costophrenic angles 


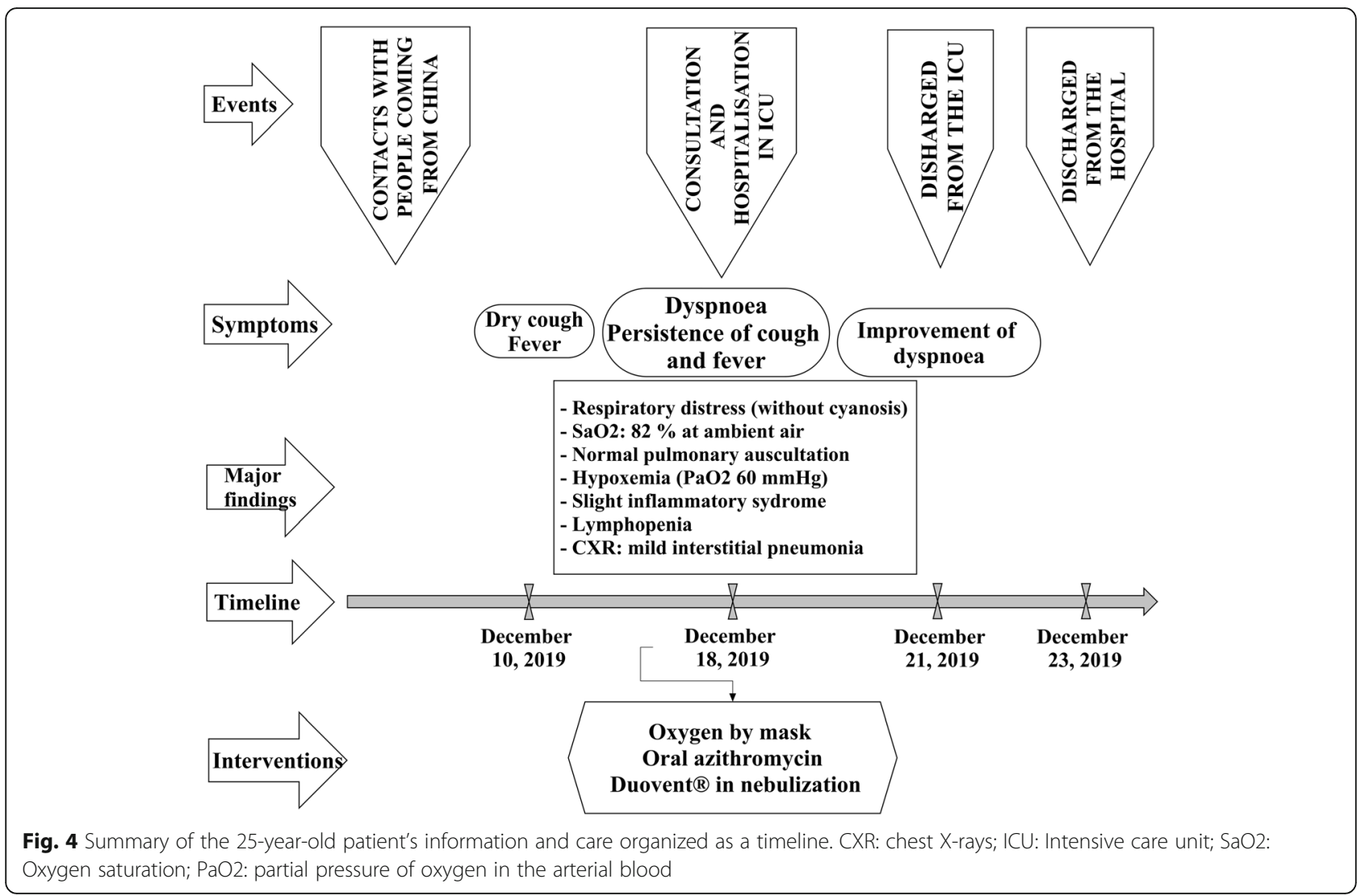

atypical causes of respiratory failure. In the second case, hypoxia was disproportionate with the chest lesions. In no case could the aetiology be accurately determined. COVID-19 was not yet known when these patients were hospitalized. However, when viewed retrospectively the two cases presented a clinical picture and laboratory changes (procalcitonin within the normal range, slight inflammation, and lymphopenia) compatible with a viral infection. In both cases, alkalosis was present as also found by others in COVID-19 patients [27]. The chest $\mathrm{X}$-ray series of the first patient showed lesions similar to those found in COVID-19 [28]. In addition, unlike the 25 -year-old female patient who had no comorbidity, the 55 -year-old male patient who had hypertension as comorbidity, developed a more severe acute respiratory distress which progressed to death. For COVID-19, available data show that older age and comorbidities such as cardiovascular diseases, diabetes, hypertension, chronic respiratory diseases and cancer are associated with an increased risk of death $[29,30]$.

The other most common cause of acute respiratory distress syndrome due to viral infections is influenza. Previous data by others suggest that ground glass opacities are less common in influenza [31]. The ground glass lesions detected in our patients are indeed more compatible with COVID-19, but it should be recognized that such radiological findings are not pathognomonic and do not allow to exclude non-COVID-19 infections. Compared to COVID-19, influenza is more frequently associated with productive cough and with less gastrointestinal symptoms [32]. Cough was indeed nonproductive in our patients, but there was no report of gastro-intestinal symptoms or of loss of taste and/or smell to more strongly support the possibility of COVID-19. A diagnosis of psittacosis can hardly be sustained in view of the dramatic evolution to death despite the use of antibiotics, including a macrolide and a quinolone, which are known to be efficacious against Chlamydophila psittaci [33]. In retrospect, especially given the context of travel to China or of contact with travellers from China, the question can thus be raised as to whether these cases could have been COVID-19 infections.

The two cases presented here are illustrative of (unpublished) observations made by clinicians in Bukavu during the last term of 2019. During that period, an abnormally high number of patients presented to local hospitals with symptoms of cough and fever, which were diagnosed as flu or atypical pneumonia for those who could have a chest radiography.

The COVID-19 epidemic has apparently been late to hit Africa, relative to the starting dates in Asia or 
Europe. This is not due to lack of contacts with China, known to be the origin of the pandemic. During the past two decades business exchanges between many regions of Africa, especially the eastern part of the continent, with Asia, and in particular with China, have been on the increase [34-36]. Even after the official declaration of the pandemic, many persons from Africa continued to travel to China, to secure the import of various goods. The apparent delay in the outbreak of COVID-19 cannot therefore be explained solely on the grounds that there is a low level of traveling between Africa and China [17, 36].

In addition, despite the evidence of community spread of COVID-19 in DRC since nearly 1 year, the progression of the number of such cases has been relatively slower compared to Europe, the USA, and Latin America. One reason for the small number of reported positive cases could arguably be due to the low level of testing. However, indirect evidence of the local propagation of the disease consists in the occurrence of an increased number of people consulting for cough, fever, dyspnoea and eventually for severe respiratory distress requiring respiratory assistance as it has been the case from the last week of May 2020 up to mid-July 2020 [22]. From mid-March 2020 when the first COVID-19 were reported in South-Kivu up to end-May 2020, such an increased incidence of cases of acute respiratory distress was not observed locally. The question is to which extent potential mechanisms underlying the relative protection involve factors such as the generally young age of the population, other immunizations (Bacille Calmette-Guérin vaccination, measles, etc.), some environmental or genetic factors, etc.

In view of the above-mentioned observations, it would also be tempting to raise the question as to whether SARS-CoV-2 infection might have already been present in the region prior to the officially declared start in China in December 2019 and account for the fact that the disease did not develop into an epidemic for so many months. Reports of COVID-19 cases before the declaration of the pandemic exist [37]. It is important to stress the limitations of using retrospective case reports (especially limited to only 2 patients) as evidence of suspected COVID-19, especially in contexts such as ours with limited detailed information on travel/contact history and with limited conservation of blood or other samples for retrospective laboratory analysis.

\section{Conclusion}

This case report highlights the fact that clinical cases presenting like COVID-19 were present in our part of eastern DRC months before the official start of the pandemic. This raises the question as to whether prior infection with SARS-Cov2 or by other infections causing cross-immunity could explain the late and smaller extent of the COVID-19 pandemic in the region. We wish to carry out larger studies, including retrospective microbiological and serological investigations in local communities in order to test this hypothesis. There is also a need to develop more robust epidemiological surveillance systems in Africa, sensitive to the threat of pandemics as a consequence of increased international traffic and globalisation.

\section{Abbreviations \\ ABG: Arterial blood gas; COVID-19: The novel coronavirus disease 2019; CRP: C-reactive protein; CXR: Chest X-Rays; DRC: The Democratic Republic of the Congo; HIV: Human Immunodeficiency Virus; HPGRB: Hôpital Provincial Général de Référence de Bukavu; ICU: Intensive Care Unit; $\mathrm{PaO}_{2}$ : Partial pressure of oxygen in the arterial blood; $\mathrm{SaO}_{2}$ : Oxygen saturation; SARS-CoV- 2: Severe acute respiratory syndrome coronavirus 2}

\section{Acknowledgements}

Not applicable.

\section{Authors' contributions}

All authors contributed to the study conception and design. GQM, PM, SM $\mathrm{AB}$, and $\mathrm{BF}$ contributed to the clinical patient care and management. Chest $X$-rays were performed and interpreted by FN and GM. All authors contributed to the literature search. The first draft of the manuscript was written by $\mathrm{MB}$ and PM and all authors contributed to subsequent versions of the manuscript. All authors read and approved the final manuscript.

\section{Funding}

There was no funding source for this study.

\section{Availability of data and materials}

All data generated or analysed during this study are included within the article.

\section{Declarations}

Ethics approval and consent to participate

All patients admitted in the "Hôpital Provincial Général de Référence de Bukavu" consent that their clinical and imaging data can be used for publication or education purpose. All procedures performed were in accordance with the ethical standards of the institutional ethical committee and with the 1964 Helsinki declaration and its later amendments.

All patients admitted consent that their clinical and imaging data can be used for publication or education purpose.

\section{Consent for publication}

Written informed consent for publication of the clinical details and/or clinical images was obtained from the patients or the next of kin (for the deceased patient).

\section{Competing interests}

The authors declare that they have no competing interests.

\section{Author details}

'Department of Internal Medicine, Hôpital Provincial Général de Référence de Bukavu (HPGRB), Bukavu, Democratic Republic of the Congo. ${ }^{2}$ Faculty of Medicine, Université Catholique de Bukavu (UCB), Bukavu, Democratic Republic of the Congo. 'École Régionale de Santé Publique (ERSP), Université Catholique de Bukavu (UCB), Bukavu, Democratic Republic of the Congo.

Received: 25 November 2020 Accepted: 23 March 2021

Published online: 06 April 2021

\section{References}

1. Zhu N, Zhang D, Wang W, Li X, Yang B, Song J, et al. A novel coronavirus from patients with pneumonia in China, 2019. N Engl J Med. 2020;382(8): 
727-33. Available from: https://pubmed.ncbi.nlm.nih.gov/31978945. https:// doi.org/10.1056/NEJMoa2001017.

2. World Health Organization (WHO). Coronavirus disease ( COVID- 19 ): Similarities and differences with influenza. Q\&A (17 March 2020). (Accessed on 25 Oct 2020). Available from: https://www.who.int/news-room/q-a-deta il/coronavirus-disease-covid-19-similarities-and-differences-with-influenza

3. Jung C-Y, Park H, Kim DW, Choi YJ, Kim SW, Chang TI. Clinical characteristics of asymptomatic patients with COVID-19: a Nationwide cohort study in South Korea. Int J Infect Dis. 2020;99:266-8Available from: https://pubmed. ncbi.nlm.nih.gov/32771632. https://doi.org/10.1016/j.ijid.2020.08.001.

4. Huang C, Wang Y, Li X, Ren L, Zhao J, Hu Y, et al. Clinical features of patients infected with 2019 novel coronavirus in Wuhan, China. Lancet. 2020;395(10223):497-506. https://doi.org/10.1016/S0140-6736(20)30183-5.

5. Wang D, Hu B, Hu C, Zhu F, Liu X, Zhang J, et al. Clinical characteristics of 138 hospitalized patients with 2019 novel coronavirus-infected pneumonia in Wuhan, China. JAMA. 2020;323:1061-9. https://doi.org/10.1001/jama.202 0.1585 .

6. Chen N, Zhou M, Dong X, Qu J, Gong F, Han Y, et al. Epidemiological and clinical characteristics of 99 cases of 2019 novel coronavirus pneumonia in Wuhan, China: a descriptive study. Lancet. 2020;395(10223):507-13. https:// doi.org/10.1016/S0140-6736(20)30211-7.

7. Guan W, Ni Z, Hu Y, Liang W, Ou C, He J, et al. Clinical characteristics of coronavirus disease 2019 in China. N Engl J Med. 2020;382(18):1708-20. https://doi.org/10.1056/NEJMoa2002032.

8. Chan JF-W, Yuan S, Kok K-H, To KK-W, Chu H, Yang J, et al. A familial cluster of pneumonia associated with the 2019 novel coronavirus indicating person-to-person transmission: a study of a family cluster. Lancet. 2020; 395(10223):514-23. https://doi.org/10.1016/S0140-6736(20)30154-9.

9. Mao L, Jin H, Wang M, Hu Y, Chen $S$, He Q, et al. Neurologic manifestations of hospitalized patients with coronavirus disease 2019 in Wuhan, China. JAMA Neurol. 2020;77:683-90. https://doi.org/10.1001/jamaneurol.2020.1127.

10. Vaira LA, Salzano G, Deiana G, De Riu G. Anosmia and Ageusia: common findings in COVID-19 patients. Laryngoscope. 2020;130(7):1787. https://doi. org/10.1002/lary.28692.

11. Gerkin RC, Ohla K, Veldhuizen MG, Joseph PV, Kelly CE, Bakke AJ, et al. The best COVID-19 predictor is recent smell loss: a cross-sectional study. medRxiv. 2020;2020.07.22.20157263 Available from: http://medrxiv.org/ content/early/2020/07/28/2020.07.22.20157263.abstract.

12. Gerkin RC, Ohla K, Veldhuizen MG, Joseph PV, Kelly CE, Bakke AJ, et al. Recent smell loss is the best predictor of COVID-19 among individuals with recent respiratory symptoms. Chem Senses. 2020. https://doi.org/10.1093/ chemse/bjaa081.

13. Wang J, Jiang M, Chen X, Montaner LJ. Cytokine storm and leukocyte changes in mild versus severe SARS-CoV-2 infection: review of 3939 COVID19 patients in China and emerging pathogenesis and therapy concepts. J Leukoc Biol. 2020;108(1):17-41Available from: https://pubmed.ncbi.nlm.nih. gov/32534467. https://doi.org/10.1002/JLB.3COVR0520-272R.

14. World Health Organization. WHO Coronavirus Disease (COVID-19) Dashboard (updated: 2021/2/22, 2:15pm CET). [Accessed on 22 Feb 2021]. Available from: https://covid19.who.int/

15. Johns Hopkins University. COVID-19 Dashboard by the Center for Systems Science and Engineering (CSSE) at Johns Hopkins University (JHU). Johns Hopkins Coronavirus Resour. Cent. [Accessed on 22 Feb 2021]. Available from: https://coronavirus.jhu.edu/map.html

16. Velavan TP, Meyer CG. The COVID-19 epidemic. Trop Med Int Health. 2020 25(3):278-80. https://doi.org/10.1111/tmi.13383.

17. Gilbert M, Pullano G, Pinotti F, Valdano E, Poletto C, Boëlle $P$, et al. Preparedness and vulnerability of African countries against importations of COVID-19 : a modelling study. Lancet. 2020;395(10227):871-7. https://doi. org/10.1016/S0140-6736(20)30411-6

18. Moore M, Gelfeld B, Okunogbe A, Paul C. Identifying future disease hot spots: infectious disease vulnerability index. Rand Heal Q. 2017;6:5 Available from: https://pubmed.ncbi.nlm.nih.gov/28845357.

19. World Health Organization (WHO). Coronavirus disease (COVID-2019) Situation report 26. 2020 [Accessed on 16 Jun 2020]. Available from: https:// www.who.int/emergencies/diseases/novel-coronavirus-2019/situationreports

20. Kapata N, thekweazu C, Ntoumi F, Raji T, Chanda-Kapata P, Mwaba P, et al. Is Africa prepared for tackling the COVID-19 (SARS-CoV-2) epidemic. Lessons from past outbreaks, ongoing pan-African public health efforts, and implications for the future. Int J Infect Dis. 2020;93:233-6. Available from: https://pubmed.ncbi.nlm.nih.gov/321 19980. https://doi.org/10.1016/j.jij.202 0.02 .049 .

21. Comité Multisectoriel de la Riposte à la Pandémie du Covid-19 en RDC. Situation épidémiologique COVID-19 en République Démocratique du Congo au 19 février 2021. [Accessed on 22 Jun 2020]. Available from: https://us3.campaign-archive.com/?u=b34a30571d429859fb249533d\&id= d7427d9e8c

22. Division Provinciale de la Santé Province du Sud-Kivu. Profil épidémiologique de la Province du Sud-Kivu. 20 février 2021.

23. Njenga MK, Dawa J, Nanyingi M, Gachohi J, Ngere I, Letko M, et al. Why is there low morbidity and mortality of COVID-19 in Africa? Am J Trop Med Hyg. 2020;103(2):564-9. https://doi.org/10.4269/ajtmh.20-0474.

24. Tso FY, Lidenge SJ, Peña PB, Clegg AA, Ngowi JR, Mwaiselage J, et al. High prevalence of pre-existing serological cross-reactivity against severe acute respiratory syndrome coronavirus-2 (SARS-CoV-2) in sub-Saharan Africa. Int J Infect Dis. 2021;102:577-83Available from: https://pubmed.ncbi.nlm.nih. gov/33176202. https://doi.org/10.1016/j.ijid.2020.10.104.

25. Mbow M, Lell B, Jochems SP, Cisse B, Mboup S, Dewals BG, et al. COVID-19 in Africa: dampening the storm? Science. 2020:369:624 LP-626 Available from: http://science.sciencemag.org/content/369/6504/624.abstract.

26. O'Neal JD, Golden MR, Branson BM, Stekler JD. HIV nucleic acid amplification testing versus rapid testing: it is worth the wait. Testing preferences of men who have sex with men. J Acquir Immune Defic Syndr. 2012;60(4):e117-20. https://doi.org/10.1097/QAl.0b013e31825aab51.

27. Bezuidenhout MC, Wiese OJ, Moodley D, Maasdorp E, Davids MR, Koegelenberg CF, et al. Correlating arterial blood gas, acid-base and blood pressure abnormalities with outcomes in COVID-19 intensive care patients. Ann Clin Biochem. 2021;58(2):95-101. https://doi.org/10.1177/000456322 0972539.

28. Jacobi A, Chung M, Bernheim A, Eber C. Portable chest X-ray in coronavirus disease-19 (COVID-19): a pictorial review. Clin imaging 2020;64:35-42. Available from. 2020. https://doi.org/10.1016/j.clinimag.2020.04.001.

29. Chen T, Wu D, Chen H, Yan W, Yang D, Chen G, et al. Clinical characteristics of 113 deceased patients with coronavirus disease 2019 : retrospective study. BMJ. 2019:368:m1091.

30. Guan W, Liang W, Zhao Y, Liang H, Chen Z, Li Y, et al. Comorbidity and its impact on 1590 patients with Covid-19 in China : a Nationwide analysis. Eur Respir J. 2020;55(5):2000547. https://doi.org/10.1183/13993003.00547-2020.

31. Cao B, Li X-W, Mao Y, Wang J, Lu H-Z, Chen Y-S, et al. Clinical features of the initial cases of 2009 pandemic influenza a (H1N1) virus infection in China. N Engl J Med. 2009;361(26):2507-17. https://doi.org/10.1056/NEJMoa 0906612.

32. Tang X, Du R-H, Wang R, Cao T-Z, Guan L-L, Yang C-Q, et al. Comparison of hospitalized patients with ARDS caused by COVID-19 and H1N1. Chest. 2020;158(1):195-205. https://doi.org/10.1016/j.chest.2020.03.032.

33. Stewardson AJ, Grayson ML. Psittacosis. Infect Dis Clin N Am. 2010;24(1):725. https://doi.org/10.1016/j.idc.2009.10.003.

34. Zhou Y. Air traffic between China and Africa has jumped $630 \%$ in the last decade. Quartz Africa. 2019; [Accessed on 24 Oct 2020]. Available from: https://qz.com/africa/1675287/china-to-africa-flights-jumped-630-in-the-pastnine-years/.

35. Pirie G. China and aviation in Africa: context, thrust, novelty. African Geogr Rev. 2020;39:74-89. https://doi.org/10.1080/19376812.2019.1599294.

36. Lalaoui R, Bakour S, Raoult D, Verger P, Sokhna C, Devaux C, et al. What could explain the late emergence of COVID-19 in Africa? New Microbes New Infect. 2020;38:100760 Available from: http://www.sciencedirect.com/ science/article/pii/S2052297520301128.

37. Deslandes A, Berti V, Tandjaoui-Lambotte Y, Alloui C, Carbonnelle E, Zahar $J R$, et al. SARS-CoV-2 was already spreading in France in late December 2019. Int J Antimicrob Agents. 2020;55(6):106006. Available from: http:// www.sciencedirect.com/science/article/pii/S0924857920301643. https://doi. org/10.1016/j.jjantimicag.2020.106006.

\section{Publisher's Note}

Springer Nature remains neutral with regard to jurisdictional claims in published maps and institutional affiliations. 